\title{
Hanseníase Virchowiana em paciente com neurofibromatose: um desafio diagnóstico
}

\author{
Virchowian Leprosy in a patient with neurofibromatosis: a diagnostic challenge \\ Lepra Virchowiana en paciente con neurofibromatosis: un desafío diagnóstico
}

Ana Carolina Carcará Franco de Sá Melo ${ }^{1 *}$, Anna Bheatriz Feitosa Couto ${ }^{1}$, Bárbara Fernandes de Meneses Brito ${ }^{1}$, Cícero Ferreira Lima Júnior ${ }^{1}$, Juliana Guimarães de Macau Furtado ${ }^{2}$, Maria Vitória Cunha de Azevedo ${ }^{1}$, Robert Chaves Ximenes de Morais ${ }^{1}$, Yandra Ariane Gomes Lima ${ }^{1}$, Evandra Marielly Leite Nogueira Freitas Galvão'.

\section{RESUMO}

Objetivo: Relatar o caso de um paciente portador de neurofibromatose e que evoluiu com Hanseníase Virchorwiana. Detalhamento do caso: Paciente do sexo masculino, 42 anos de idade, portador de neurofibromatose tipo 1, apresentou placas eritemato - edematosas maiores que $3 \mathrm{~cm}$ em tronco e membros superiores associado a edema de mãos e pés, espessamento do nervo ulnar direito, nódulos de Lisch na íris, madarose e infiltração de septo nasal por bacilos. Índice baciloscópio geral 2,75 para bacilos fragmentados e íntegros, confirmando o diagnóstico de Hanseníase Virchowiana e reação Hansênica tipo 1. Considerações Finais: A coexistência de hanseníase com neurofibromatose é um achado raro e pode representar um dilema diagnóstico, tendo em vista a semelhança clínica entre as duas doenças. $E$, ambas ligadas a reação hansênica tipo I, formam um conjunto de distúrbios que afetam as células de Schwann. Nesse âmbito, ainda são necessárias realizações de mais pesquisas para uma melhor avaliação entre a associação de neurofibromatose e hanseníase.

Palavras-chave: Neurofibromatose, Hanseníase, Reação Hansênica.

\begin{abstract}
Objective: To report the case of a patient with neurofibromatosis who developed Virchorwian Leprosy. Case details: Male patient, 42 years old, with type 1 neurofibromatosis, presented erythematous plaques edematous greater than $3 \mathrm{~cm}$ on the trunk and upper limbs associated with edema of the hands and feet, thickening of the right ulnar nerve, nodules of Lisch in the iris, madarosis and infiltration of the nasal septum by bacilli. General bacilloscope index 2.75 for fragmented and intact bacilli, confirming the diagnosis of Virchowian leprosy and type 1 leprosy reaction. Final Considerations: The coexistence of leprosy with neurofibromatosis is a rare finding and may represent a diagnostic dilemma, given the clinical similarity between the two diseases. And, both linked to type I leprosy reaction, form a set of disorders that affect Schwann cells. In this context, further research is needed to better assess the association between neurofibromatosis and leprosy.
\end{abstract}

Key words: Neurofibromatosis, Leprosy, Leprosy reaction.

\section{RESUMEN}

Objetivo: Informar el caso de un paciente con neurofibromatosis que evolucionó con Lepra Lepromatosa. Datos del caso: Paciente masculino de 42 años con neurofibromatosis tipo 1 que presenta placas eritematosas - edematosas mayores de $3 \mathrm{~cm}$ en tronco y extremidades superiores asociadas a edema de

${ }^{1}$ Centro Universitário Uninovafapi (UNINOVAFAPI), Teresina - PI. *E-mail: anacarolinacarcara@gmail.com

${ }^{2}$ Universidade Federal do Piauí (UFPI), Teresina - PI.

SUBMETIDO EM: 6/2021

ACEITO EM: 7/2021

PUBLICADO EM: 9/2021 
manos y pies, engrosamiento del nervio cubital derecho, nódulos de Lisch en el iris, madarosis e infiltración del tabique nasal por bacilos. Índice de baciloscopio general 2,75 para bacilos fragmentados e intactos, que confirma el diagnóstico de lepra de Virchow y reacción a la lepra tipo 1. Consideraciones finales: La coexistencia de lepra con neurofibromatosis es un hallazgo raro y puede representar un dilema diagnóstico, dada la similitud clínica entre las dos enfermedades. Y, ambos relacionados con la reacción a la lepra tipo I, forman un conjunto de trastornos que afectan a las células de Schwann. En este contexto, se necesitan más investigaciones para evaluar mejor la asociación entre neurofibromatosis y lepra.

Palabras clave: Neurofibromatosis, Enfermedad de Hansen, Reacción a la lepra.

\section{INTRODUÇÃO}

No ano de 2020, de acordo com o Ministério da Saúde, foram notificados 13.807 casos de hanseníase, sendo a faixa etária mais comum dos 40 aos 48 anos. A prevalência dessa patologia é bastante variável, sendo que a grande maioria das ocorrências relatadas são de países em desenvolvimento (MAYMONE M, et al., 2020; FAVA VM, et al., 2020).

A hanseníase, é uma das doenças infecciosas mais antigas, uma patologia crônica de pele e nervos periféricos, causada pelo Mycobacterium leprae (M. leprae). O meio de transmissão ainda não é completamente esclarecido e parece acontecer de forma zoonótica, tendo forte relação com a transmissão por contato interpessoal. Apesar disso, a exposição ao M. leprae não é suficiente para causar hanseníase de forma clínica, em geral, a maioria dos indivíduos acaba não desenvolvendo a doença após a exposição. Por isso, a infecção é uma combinação de fatores ambientais, de carga do patógeno e de suscetibilidade genética, que resulta ou não em seu aparecimento (MAYMONE M, et al., 2020; FAVA VM, et al., 2020).

O diagnóstico deve ser considerado em cenário de lesões de pele crônicas, que não respondem ao tratamento para uma condição clínica mais comum, ou ainda que tenha envolvimento de perda sensorial. Essa patologia é classificada em Paucibacilar e Multibacilar, sendo esta a forma que contém a Hanseníase Virchowiana (HV) que é a mais disseminada da doença. Nela, as lesões podem se apresentar como máculas, pápulas e/ou nódulos eritematosos e estão distribuídas simetricamente no corpo. Em alguns casos, se apresenta com infiltração difusa e espessamento da pele (SCOLLARD D, et al., 2020).

A neuropatia, ocasionada pela hanseníase, ocorre de forma precoce e é caracterizada por diminuição ou ausência de sensibilidade nas lesões, podendo evoluir ao curso da doença. Por conta disso, a avaliação dos nervos periféricos é essencial em todo exame físico. Além disso, diagnóstico e tratamento precoce são imprescindíveis para minimizar os danos ao paciente (SCOLLARD D, et al., 2020).

Por outro lado, as Neurofibromatoses (NF) são um grupo de doenças heterogêneas, neurogênicas hereditárias e autossômicas dominantes. Há 3 formas clínicas e geneticamente distintas de NF: a Neurofibromatose tipo 1 (NF1), Neurofibromatose tipo (NF2), e Schwannomatose (CIMINO PJ e GUTMANN $\mathrm{DH}, 2018)$.

A NF acomete cerca de 80 mil brasileiros, devido ao aumento de conhecimento científico sobre a NF há uma melhora do manejo clínico e redução da morbidade das complicações, ocasionando maior qualidade de vida para os pacientes acometidos. Apesar da NF poder ser diagnosticada na maioria das especialidades 0 acompanhamento de especialistas em NF para tratamento adequado se torna fundamental, já que há uma expressão fenotípica muito variável, curso progressivo, envolvimento de múltiplos órgãos e evolução natural imprevisível (RODRIGUES L, et al., 2014).

Dentre as formas clínicas, a Schwannomatose é a mais rara, tem prevalência de aproximadamente metade do acometimento da neurofibromatose tipo 2 (NF2), em uma população do noroeste da Inglaterra, a prevalência foi em cerca de 1 em 126.000 (YOHAY K, et al., 2021). Já a NF1 é a mais comum, com prevalência de 2500-3000 indivíduos em todo o mundo e ocorre devido a uma alteração no gene Nf1 localizado no cromossomo 17q11.2 (CIMINO PJ e GUTMANN DH, 2018). 
A NF1 apresenta um componente genético. Metade dos casos de NF1 é herdada de um dos pais, já os outros $50 \%$, não apresentam histórico familiar e sugere alta incidência de novas mutações. Essa alteração no gene tem como consequência a ausência da proteína neurofibromina, por incapacidade de síntese. Essa ausência pode ser encontrada nas lesões de NF1 como nas pseudoartroses e nos neurofribomas (KORF BR, et al., 2015).

As manifestações clínicas de NF podem ser caracterizadas pela presença de máculas café com leite, sardas axilares e/ou inguinais, nódulos de Lisch além de formação de tumores. Esses tumores são chamados de neurofibromas, que quando cutâneos são benignos e não apresentam risco de transformação maligna. Eles são móveis ao exame físico, e podem apresentar-se com prurido. O diagnóstico da NF1 é baseado na presença de características clínicas e nem sempre o teste genético é necessário para confirmá-lo, porém pode ser útil em alguns casos (KORF BR, et al., 2015).

Apesar da etiologia e da patogênese serem diferentes nas doenças descritas, suas características principais incluem o acometimento neural, sendo o alvo principal a célula de Schwann e as lesões cutâneas. A simultaneidade desses dois distúrbios pode levar à uma confusão diagnóstica, uma vez que existe uma semelhança clínica entra as duas patologias (QUYUM F, et al., 2015).

Assim, o presente manuscrito tem como objetivo relatar o caso de um paciente portador de neurofibromatose e que evoluiu com Hanseníase Virchorwiana.

\section{DETALHAMENTO DO CASO}

Paciente G.S.S, sexo masculino, 42 anos, procedente e residente da cidade de Teresina no Piauí, procurou o serviço de dermatologia em novembro de $2020 \mathrm{com}$ uma queixa de presença de placas eritematoedematosas em tronco e membros superiores há cerca de 6 meses. Referiu que as placas eritematoedematosas não apresentavam alteração de sensibilidade. Além disso, o paciente continha edema de mãos e pés, espessamento do nervo ulnar direito, madarose e infiltração do septo nasal pela presença de bacilos.

Ao exame físico, também foram observadas lesões nodulares disseminadas depressíveis ao toque, compatível com neurofibromas, além de manchas café com leite espalhadas pelo corpo e Nódulos de Lisch na íris. Durante a anamnese, previamente, o paciente confirmou o diagnóstico de NF desde os 12 anos de idade e relatou a presença de histórico familiar (mãe portadora da doença). Em adição, afirmou não ter tido contato com portadores de hanseníase.

A partir da avaliação do paciente, foi solicitada a baciloscopia, que se apresentou positiva (índice baciloscópico geral de 2,75 para bacilos fragmentados e íntegros). Com a característica da baciloscopia, aliada ao surgimento de novas lesões agudas inflamatórias foi firmada a associação diagnóstica entre HV e reação hansênica tipo 1 (grau de incapacidade 1). Após comprovação diagnóstica, houve um momento durante a consulta para esclarecimentos sobre ambas as patologias e foi iniciado tratamento com poliquimioterapia multibacilar associado a prednisona $40 \mathrm{mg}$. Em consultas seguintes, o paciente referiu melhora importante do quadro e negou o surgimento de efeitos adversos. O paciente assinou o termo de livre consentimento esclarecido (TCLE).

\section{DISCUSSÃO}

A NF1, ou doença de von Recklinghausen, é uma enfermidade de caráter autossômico dominante que contribui com $90 \%$ dos casos de NF (RALLIS E e ATHANASIOS A, 2015). A NF1 é marcada pela presença de neurofibromas, que são tumores benignos de bainhas de nervos periféricos (BERGQVIST C, et al., 2020). O gatilho inicial para formação desses tumores é a perda do alelo de neurofibrina nas células de Schwann, que leva à migração de mastócitos para dentro da lesão do neurofibroma. Isso acarreta na formação do tumor pela expressão de fator de transformação de crescimento beta (TGFß). Assim, ocorre a proliferação de células fusiformes, de células de Schwann, de mastócitos e de componentes vasculares, contribuindo para a sua formação. Eles afetam entre 30 a 50\% dos pacientes com essa doença (OZARSLAN B, et al., 2021).

Além disso, é de conhecimento científico que essa doença possui características hereditárias, sendo que, acredita-se que ela seja herdada de $50 \%$ dos pais. Os outros $50 \%$ dos casos não apresentam história familiar de NF1, sugerindo alta incidência de novas mutações (KORF BR, et al., 2015). 
Ambos os sexos podem ser acometidos e é herdada de forma autossômica dominante com penetrância completa com o gene se localizando no cromossomo 17. A NF é a doença hereditária mais frequente entre seres os seres humanos com esse padrão hereditário. Apesar de várias mutações do gene da NF1 já terem sido identificadas e estudadas, não se conseguiu concluir relações específicas entre os tipos de mutações e os vários fenótipos da doença supracitada (ANTÔNIO J, et al., 2013). Nesse contexto, é importante ressaltar que a história familiar do paciente apresenta um peso importante para seu diagnóstico de NF, visto que, a mãe também apresenta essa patologia.

A neurofibromatose constitui uma patologia com repercussão sistêmica progressiva, podendo gerar alterações no sistema nervoso central e periférico, bem como as mais diversas manifestações físicas (MORAES F, et al., 2013).

Além dos sinais e sintomas clássicos, a NF pode gerar limitações sociais ao indivíduo, tendo em vista o comprometimento da vida socioafetiva, desenvolvimento escolar e relações familiares, que são resultado das ocorrências estéticas causadas pela doença. Nessa perspectiva, a NF por ter caráter genético ocasiona em inseguranças sobre a possível transmissão da doença de forma genética e hereditária sendo mais um fator limitante para o desenvolvimento social desses pacientes (CERELLO A, et al., 2013).

O M. leprae, agente etiológico da Hanseníase, também tem predileção pelo tecido neural. Dessa forma, a primeira evidência de infecção é, frequentemente, encontrada no sistema nervoso periférico. A amostra de pele para biópsia deve ser retirada do lado ativo da lesão e deve conter toda a espessura da derme para correta análise. Esse microorganismo é intracelular obrigatório e tem afinidade por macrófagos e células de Schwann. O dano nervoso é causado pela infecção do bacilo no nervo periférico e pela resposta imunológica e inflamatória. O elevado estado imunológico leva ao intenso envolvimento nervoso (KUNDAKCI $\mathrm{N}$ e ERDEM C, 2019).

No acometimento cutâneo da hanseníase multibacilar, a epiderme se torna afinada, as cristas de Rete se achatam e há formação difusa de granulomas (lepromas) na derme, contendo numerosos bacilos. Tais granulomas consistem, principalmente, de macrófagos que não se convertem e de células epiteliais. Há poucos ou nenhuns linfócitos. Em estágios avançados, há a formação de células de Virchow através de alterações no citoplasma dos macrófagos e ocorre inflamação perineural dos nervos cutâneos. $O$ reconhecimento da hanseníase se dá, principalmente, por: lesões de pele hipopigmentadas ou eritematosas com perda de sensibilidade, envolvimento de nervos periféricos com espessamento e perda de sensibilidade e esfregaço cutâneo positivo para bacilos álcool-ácido resistentes (KUNDAKCI N e ERDEM C, 2019).

Reações Hansênicas são reflexos sistêmicos decorrentes de inflamação aguda proporcionada pela ação do sistema imunológico do hospedeiro ao atacar o bacilo, assim, tem por principais características típicas sinais flogísticos no local de atuação do bacilo e perda da função (BRASIL, 2017).

Dessa forma, estas se caracterizam por consequências da doença as quais afetam, especialmente, nervos e pele, o que pode ocasionar desde sequelas relevantes a morbidade. Assim, podem-se classificar em dois tipos: tipo 1 e tipo 2 (LASTÓRIAL JC e ABREU M, 2012).

Além disso, dentre as características dessa complicação imunológica, têm-se edema significativo de membros inferiores e presença de eritema significativo. Nesse contexto, foi observada a presença desses sintomas no paciente, mesmo após o tratamento poliquimioterápico inicial, o que corroborou a característica de reação hansênica, contudo a permanência no tratamento é de extrema importância na recidiva dos sintomas. Em geral, pacientes com diagnóstico de hanseníase virchowiana tendem a manifestar reações tardiamente ao tratamento com poliquimioterapia, como aconteceu no caso em estudo. Na literatura, ainda, não há um esclarecimento acerca da associação entre a permanência de neurofibromas e a predisposição do paciente de manifestar reação hansênica (BRASIL, 2017).

Mediante a escassez de conteúdo científico para uma doença milenar, da falta de padronização na definição de critérios diagnóstico e de tempo de seguimento, tem-se que, atualmente, no que se refere ao tratamento de Hanseníase, a poliquimioterapia desenvolve um resultado mais positivo na melhora clínica se comparado a outros métodos de terapia, a exemplo da combinação entre rifampicina-ofloxacina-minociclina (LAZO-PORRAS M, et al., 2020). 
A NF pode ser associada com a hanseníase tuberculoide (paucibacilar), com a históide-símile e com a lepromatosa (multibacilar). No entanto, a coinfecção com a forma multibacilar é pouco relatada na literatura (KARTHIKEYAN K, et al., 2012). A Hanseníase pode ser confundida com a NF, principalmente, devido ao acometimento neural, que é comum a ambas as doenças. É importante se atentar a suspeita de hanseníase não só em áreas endêmicas, como também em áreas não endêmicas, para que o diagnóstico seja dado de forma precoce e o tratamento correto não seja negligenciado (QUYUM F, et al., 2015)

O reconhecimento da hanseníase mostra-se importante, pois além de ter alto poder de incapacitação física, pode desencadear agravos biopsicossociais devido à apresentação de deformidades, lesões dermatológicas e isolamento e exclusão social forçados por causa da sua estigmatização (RIBEIRO L, et al., 2021). Nesse contexto, a doença pode ser um obstáculo na realização das atividades diárias, sociais e no trabalho, impactando na geração de renda e, consequentemente, no bem-estar individual e familiar. Concomitantemente, com a identificação e tratamento precoce da doença, é possível evitar a disseminação para a comunidade, além de ser oportunidade para a promoção em saúde naquela área sobre a patologia em questão e prevenir deficiências ou incapacidades físicas no paciente (BRASIL, 2017).

Portanto, considera-se que a coexistência de hanseníase com a NF é um achado bem raro e pode representar um dilema diagnóstico para o médico, visto que algumas de suas características clínicas são semelhantes. A Neurofibromatose coexistindo com a HV e reação hansênica tipo 1 não foi relatada anteriormente na literatura. Além disso, a etiologia e a fisiopatologia dessas doenças são diferentes, sendo a hanseníase uma infecção pelo M.leprae e neurofibromatose sendo uma genodermatose. Ambos os distúrbios afetam as células de Schwann e seus méritos de relacionamento ainda merecem mais estudos.

\section{REFERÊNCIAS}

1. ANTÔNIO J, et al. Neurofribromatose: hitórico cronológico e aspectos atuais. Anais Brasileiros de Dermatologia, 2013; 88(3): 333-347.

2. BERGQVIST C, et al. Neurofibromatosis 1 French national guidelines based on an extensive literature review since 1966. Orphanet Journal of Rare Diseases, 2020; 15(1): 1-23.

3. BRASIL. 2017. Ministério da Saúde. Guia Prático sobre a Hanseníase.

4. CERELLO A, et al. Representações sociais de pacientes e familiares sobre a neurofibromatose tipo 1. Ciência \& saúde coletiva, 2013; 18(8): 2362-2364

5. CIMINO PJ, GUTMANN DH. Neurofibromatosis type 1. Handbook of Clinical Neurology, 2018; 148: $799-811$.

6. FAVA VM, et al. Genetics of leprosy: today and beyond. Human genetics, $2020 ; 139(6): 835-846$.

7. KARTHIKEYAN K, et al. Double trouble: A case of von Recklinghausen's disease with coexistent lepromatous leprosy. Journal of Infection in Developing Countries, 2012; 6(3): 287-289.

8. KORF BR, et al. Neurofibromatosis type 1 (NF1): Pathogenesis, clinical features, and diagnosis. UpToDate, 2015.

9. KUNDAKCI N, ERDEM C. Leprosy: A great imitator. Clinics in Dermatology, 2019; 37(3): 200-212.

10. LASTORIAL JC, ABREU M. Hanseníase: Diagnóstico e Tratamento. Diagnóstico \& Tratamento, 2012; $17(4): 173-9$.

11. LAZO-PORRAS $\mathrm{M}$, et al. World Health Organization (WHO) antibiotic regimen against other regimens for the treatment of leprosy: a systematic review and meta-analysis. BMC Infectious Diseases, 2020; 20(1): 1 -14.

12. MAYMONE M, et al. Leprosy: Clinical aspects and diagnostic techniques. J AM ACAD DERMA, 2020; 83(1): 1-14.

13. MORAES F, et al. Neurofibromatose tipo 1. Revista brasileria de oftamologia, 2013; 72 (2): 129-131.

14. OZARSLAN B, et al. Cutaneous findings in neurofibromatosis type 1. Cancers, 2021; 13(3): 1-17.

15. QUYUM F, et al. A case of neurofibromatosis type1 with coexisting borderline tuberculoid leprosy. Journal of Pakistan Association of Dermatologists, 2015; 25(2): 136-139

16. RALLIS E, ATHANASIOS A. Exophytic Growths on the Back. American Family Physician Jour, 2015; 91 (7): $487-488$.

17. RIBEIRO L, et al. Características demográficas e clínicas do grau de incapacidade física associadas ao diagnóstico e alta do tratamento da hanseníase. Revista Eletrônica Acervo Saúde, 2021; 13(2): e6008,

18. RODRIGUES L, et al. Neurofibromatoses: part 1 - diagnosis and differential diagnosis. Arq Neuropsiquiatr. $2014 ; 72$ : 241-250.

19. SCOLLARD D, et al. Leprosy: Epidemiology, microbiology, clinical manifestations, and diagnosis. UpToDate, 2020.

20. YOHAY K, et al. Schwannomatose. UpToDate, 2021. 